\title{
Motor Imagery in Mental Rotation: An fMRI Study
}

\author{
Guy Vingerhoets,* Floris P. de Lange,* Pieter Vandemaele, $\uparrow$ Karel Deblaere, $\dagger$ and Erik Achten $†$ \\ *Laboratory for Neuropsychology, Department of Internal Medicine-Section Neurology and \\ †Department of Radiology, Ghent University, Belgium
}

Received February 25, 2002

Twelve right-handed men performed two mental rotation tasks and two control tasks while whole-head functional magnetic resonance imaging was applied. Mental rotation tasks implied the comparison of different sorts of stimulus pairs, viz. pictures of hands and pictures of tools, which were either identical or mirror images and which were rotated in the plane of the picture. Control tasks were equal except that stimuli pairs were not rotated. Reaction time profiles were consistent with those found in previous research. Imaging data replicate classic areas of activation in mental rotation for hands and tools (bilateral superior parietal lobule and visual extrastriate cortex) but show an important difference in premotor area activation: pairs of hands engender bilateral premotor activation while pairs of tools elicit only left premotor brain activation. The results suggest that participants imagined moving both their hands in the hand condition, while imagining manipulating objects with their hand of preference (right hand) in the tool condition. The covert actions of motor imagery appear to mimic the "natural way" in which a person would manipulate the object in reality, and the activation of cortical regions during mental rotation seems at least in part determined by an intrinsic process that depends on the afforded actions elicited by the kind of stimuli presented. 2002 Elsevier Science (USA)

\section{INTRODUCTION}

Whereas perception occurs when information is registered directly from the senses, mental imagery occurs when perceptual information is assessed from memory (Kosslyn et al., 2001a). The existence of mental "images," whether visual, acoustic, or motor in nature, has been a controversial issue. Some regard imagery as an epiphenomenal experience of the linguistic mental descriptions that constitute thought (Pylyshyn, $1973,1981)$. The first to show convincingly that the concept of mental imagery was amenable to scientific inquiry were Shepard and Metzler (1971). They found that when people compare two similar objects at different orientations, an increment of response time is required for each degree of angular disparity between the objects. The results suggest that the decision process involves the forming of a visual image of an object and mentally rotating the image until it is congruent with the comparison object. These and similar findings have been replicated in a wealth of experiments (for a review, see Kosslyn, 1994; Shepard and Cooper, 1982), and the general interpretation of the mental rotation process as evidence of a nonverbal form of thought process is agreed.

Recent neuroimaging research focused on the functional neuroanatomy of mental rotation. What can be concluded from these studies is that in mental rotation a host of processes is involved rather than one specific area. Three brain complexes are commonly hypothesized to be involved in the mental rotation process: (1) superior parietal cortical regions, (2) extrastriate visual regions, and (3) motor and/or premotor regions. Most studies examining mental rotation by use of functional magnetic resonance imaging ( $\mathrm{fMRI}$ ) or positron emission tomography (PET) find activation in an area comprising the superior parietal lobule (SPL) and the intraparietal sulcus (IPS) (Alivesatos and Petrides, 1997; Harris et al., 2000; I waki et al., 1999; J ordan et al., 2001; Richter et al., 1997, 2000). Several studies report a linearly increasing bilateral activity in the IPS with greater angular disparity or difficulty (Carpenter et al., 1999; Tagaris et al., 1996). With most research results being consistent in this matter, it is plausible to assume a role for SPL and IPS as a computation center for spatial transformations such as mental rotation.

Mental imagery has been metaphorically described as "seeing with the mind's eye." During mental transformation of an object linearly or rotationally, Brodmann's Area (BA) 19 (V5), an area known to respond to the perception of motion (Howard et al., 1995), is significantly activated (Barnes et al., 2000). Cohen et al. (1996) also find activation in V5 (and sometimes in BA 18) in a mental rotation task and suggest that this area is a processing center for object movement.

I maging studies are not entirely consistent in their results as to whether motor processes are involved in mental rotation. In a PET study Cohen et al. (1996) 
report activation in BA 6, a premotor area, in half of the subjects in a mental rotation task using the Shepard and Metzler (1971) cube figures. Richter et al. (2000) found bilateral premotor cortex and supplementary motor area activation using the same paradigm in a time-resolved single-trial fMRI study. The authors observe that the nonprimary motor areas were active during the mental rotation process, while the left primary motor cortex (M1) was activated at the end of each trial, reflecting activation caused by the mouse button press with the right hand at the end of each trial. In contrast, rotation studies using letter or hand stimuli regularly detect precentral gyrus activation that is unlikely to reflect simple button press activity (Kosslyn et al., 1998; Tagaris et al., 1996; Vingerhoets et al., 2001). Other studies using the same or very similar rotation paradigms, although consistent in finding activation in SPL and sometimes visual-perceptual areas, do not find premotor or motor activation (Barnes et al., 2000; Harris et al., 2000; J ordan et al., 2001).

Several strands of indirect evidence contribute to the idea that motor processes guide mental rotation. Wexler et al. (1998) showed that when participants performed a mental rotation task while executing an unseen motor rotation in a given direction at a previously learned speed, motor rotation compatible with mental rotation resulted in faster responses and fewer errors than when the two rotations were incompatible. This seems to be the case for the mental manipulation of images of objects that are commonly grasped by hand such as a screwdriver (deSperati and Stucchi, 1997). Another finding that suggests a link between physical rotation and mental rotation comes from a study by Parsons (1994) in which participants had to identify a picture as being of a left or a right hand. Reaction times were higher when the pictures had to be mentally rotated in a way that would be awkward to perform physically. Third, Georgopoulos et al. (1989), using direct cell registration in monkeys, found that neurons in the motor cortex discharged before a monkey began to shift a lever and that neurons that were tuned for orientations near the starting point of the lever fired first, followed by neurons tuned for adjacent orientations, and so on. However, this mental transformation was always preparation for an overt movement and thus cannot be generalized to a pure mental transformation (i.e., not followed by overt movement), as is the case in mental rotation.

One possible cause of the differences in activation of the brain's (pre)motor areas may be the experimental paradigm that is used in these studies or, more precisely, the task stimuli equipped. Kosslyn et al. (1998) examined the influence of stimulus material on the neural mechanisms of mental rotation by comparing mental rotation of hands to mental rotation of cube figures in a PET study. Mental rotation of cubes led to activation in the parietal lobe and BA 19, a higher perceptual visual area, while mental rotation of hands engendered activation in the left precentral gyrus (M1), SPL and inferior parietal lobule (IPL), primary visual cortex, insula, and left frontal areas BA 6 and BA 9. While not all of these differences in neural activation can be readily attributed to the differences in task stimuli, it is striking that mental rotation of hands recruited processes that prepared motor movements while mental rotation of cube figures did not. Kosslyn et al. (1998) supposed the existence of two distinct mechanisms that can be called upon in mental rotation: an internal strategy in which one anticipates what one would see if he/she were to physically manipulate the objects and an external strategy in which one visualizes the consequences of someone else or an external force moving the object. The internal strategy may have been used in the hand condition because participants were mentally preparing to move their hands. In this case, the imagery focused on a mental motor transformation of the viewer rather than of the viewed object. The external strategy may have been called upon in the cube condition, as this kind of stimulus does not prime one to move ones own hands and thus does not involve motor processes. The findings of Kosslyn et al. (1998) are not backed up by all research, as some other studies, equipped with the same Shepard-Metzler cubes, did find motor and/or premotor activation (Cohen et al., 1996; Richter et al., 2000). An explanation for these apparently contradictory re sults is that the internal strategy is not solely used when mental rotation involves hands but that it is also employed when the object lends itself to being mentally grasped and moved. Three-dimensional ShepardMetzler cubes are abstract shapes, nonexistent in the real world and therefore difficult to mentally grasp and manipulate. This may lead to a higher susceptibility to using the external strategy; not ruling out that (some) participants could use the internal strategy. This hypothesis is confirmed in a PET study in which volunteers were explicitly instructed to use an internal or external strategy in a paradigm using ShepardMetzler cubes (Kosslyn et al., 2001b). When the subjects imagined objects rotating as a consequence of physically turning them, primary motor cortex and other motor areas were activated; when they imagined objects rotating as a consequence of an external force (an electric motor), the primary motor cortex was not activated. The premotor cortex was activated in both cases, indicating that some more abstract motor processes were involved in every mental rotation, regardless of the strategy selected. It appears from these findings that subjects can voluntarily adopt one or the other strategy by request, but also that in a free-choice paradigm the preferred strategy appears to be stimulus dependent. If this is the case, participants confronted with objects that are less abstract and more 
frequently manipulated by hand (e.g., a can opener) would be more inclined to use the internal strategy.

The aim of the present study is to clarify the role of the graspability of task stimuli in possibly different pathways of mental rotation. We designed two types of stimuli: pictures of hands and pictures of tools frequently manipulated by hand. We hypothesize that the internal strategy is not confined to body parts, such as pictures of hands, and that pictures of tools frequently manipulated by hand also will preferentially trigger the internal strategy. In the neural domain we expect that both stimulus types will share a common neural substrate, namely involvement of the superior parietal cortex and extrastriate visual regions. In addition we expect the recruitment of preparatory motor processes in the condition where participants need to mentally rotate pictures of hands or tools as they are expected to trigger the internal strategy.

\section{MATERIALS AND METHODS}

\section{Participants}

Thirteen normal male volunteers participated in this experiment following written informed consent according to the institutional guidelines of the Ethics Committee of the Ghent University Hospital. One participant reported claustrophobia during the experiment, after which scanning was stopped immediately. Data from this participant were not used for subsequent analysis. All participants were consistent right-handers as measured by the Edinburgh Handedness I nventory (laterality index, mean \pm SD, $0.89 \pm 0.12$; range, 0.66-1.00) (Oldfield, 1971) and between 19 and 49 years of age (mean \pm SD, $29 \pm 9$ years). Prior to scanning, the participants were trained in the rotation task with a block of practice trials that were not used during the experiment. All participants reported that they were able to perform the task without difficulty. The participants were not aware of the purposes or predictions of the experiment until after test completion.

\section{Materials}

Two types of pictures were constructed: (1) realistic pictures of hands and (2) realistic pictures of tools. Four pictures of hands were constructed with a digital camera (Kodak DC290 Zoom) and picture-editing software (Adobe PhotoShop 5). We used hands in different orientations (e.g., making the victory symbol, all five fingers raised, etc.). Four pictures of tools were constructed in the same way. The tools used were a pencil sharpener, a soup ladle, a can opener, and a monkey wrench. They were asymmetric in all three dimensions. All pictures were scaled to the same proportions and grayscaled in order to obtain the same visual load for all types of stimuli.
Stimuli were constructed with these pictures. Each stimulus consisted of a pair of pictures that were either the same or mirror images of each other. In the control condition, the angular disparity between the figures was $0^{\circ}$. Four orientations of these pictures were used $\left(0,90,180\right.$, and $\left.270^{\circ}\right)$, half of the pictures had the same pairs of figures, and half of them had mirror-image pairs of figures. The mirror-image pairs were aligned so that the figures did not differ in the angle of rotation. Further, half of the hand stimuli had a left hand in the left corner of the picture, whereas the other half had a right hand in the left corner of the picture. In this way 64 different pictures were created per control condition.

Rotation of one of the figures in order to create an angular disparity between 90 and $270^{\circ}$ in incremental steps of $30^{\circ}$ yiel ded experimental stimuli. Figures were rotated in the plane of the picture. Each orientation appeared at least nine times, adding up to a total of 64 different pictures per experimental condition.

\section{Experimental Paradigm}

Each participant took part in four experimental conditions, of which two were mental rotation tasks and two their control condition counterparts (depicted in Fig. 1): a same-different judgment of (1) nonrotated figures of hands (control hands, $\mathrm{CH}$ ), (2) rotated figures of hands (experimental hands, EH), (3) nonrotated figures of tools (control tools, CT), and (4) rotated figures of tools (experimental tools, ET).

Stimuli were presented in blocks for scanning purposes because there is latency between task-related brain activity and a hemodynamic response. Each condition was divided into eight blocks, the blocks each consisting of eight stimuli. Timing was stimulus-paced, i.e., after $4 \mathrm{~s}$ a new stimulus appeared regardless of whether the participant had answered to control for visual load between conditions. Within each block, the number of same and mirror image stimuli as well as the number of right hands and left hands was equal. These blocks were semirandomized so that the control condition preceded the experimental condition just as often as the experimental condition preceded the control condition and that all conditions had appeared once before a new set of blocks was presented.

Participants made same/different judgments by pressing an $\mathrm{fMRI}$-compatible button box with their left hand. Participants were instructed to lie as still as possible to prevent motion artifacts. Participants pressed the buttons with their left hand to make sure that a possible left-hemisphere motor activation could not be the result of the button press. Stimulus presentation, recording of the response times, hit rates, and errors were controlled by in-house software (P.V.). 
same
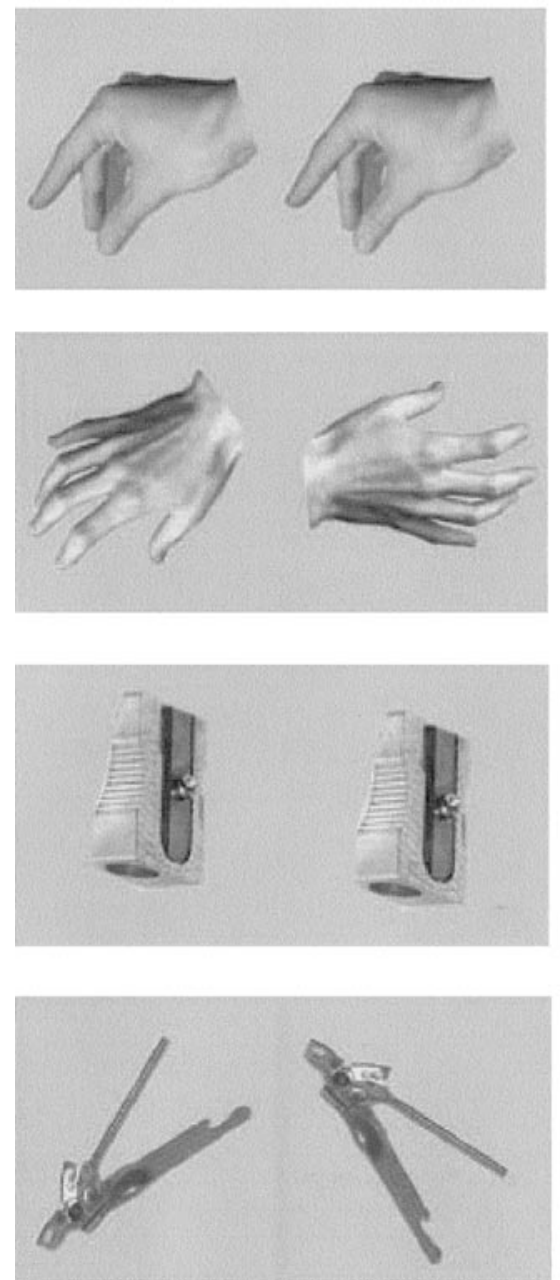

different

CH

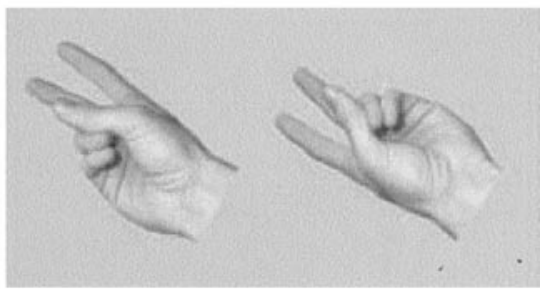

EH

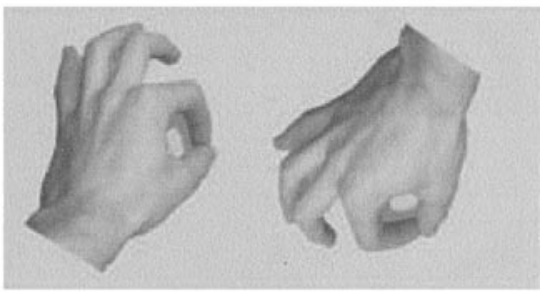

CT

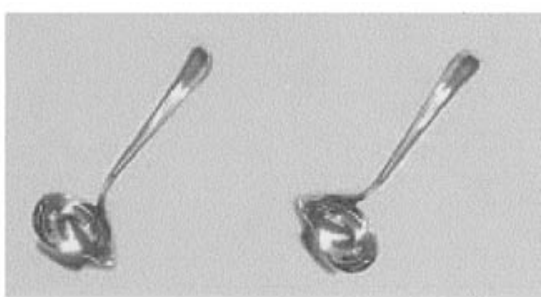

ET

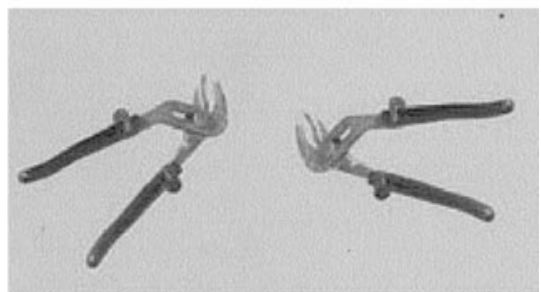

FIG. 1. Illustrations of "same" and "different" stimuli in control $(C)$ and experimental $(E)$ conditions for hands (H) and tools $(T)$.

\section{Scanning Procedure}

Participants were scanned in a Siemens Symphony 1.5-T MRI system equipped with echo planar imaging (EPI) capabilities using the standard head coil for radiofrequency transmission and signal reception. At first a series of $\mathrm{T}_{1}$-weighted anatomical images for each participant was obtained to serve as a basis for the determination of anatomical landmarks and coordinates after which the magnetic field was shimmed on each individual participant. Following this, a 3D highresolution $\mathrm{T}_{1}$ anatomical image was acquired for coregistration with the EPI images (3D MPRAGE, 128 slices, slice thickness $=1.25 \mathrm{~mm}$, in-plane resolution $=$ $0.9 \times 0.9 \mathrm{~mm}, \mathrm{TR}=2010 \mathrm{~ms}, \mathrm{TE}=5.13)$. Then, 384 EPI images were acquired during stimulus presentation. Scanning parameters of these images were TR = $4 \mathrm{~s}, \mathrm{TE}=60 \mathrm{~ms}$, flip angle $=90^{\circ}, 33$ slices, slice thickness $=5 \mathrm{~mm}, \mathrm{FOV}=192 \mathrm{~mm}$, and matrix $=64 \times$ 64 , resulting in a resolution of $3 \times 3 \times 5 \mathrm{~mm}$.

\section{I mage Analysis}

Image analysis was performed on a PC workstation using MATLAB and SPM99 software (URL http:// www.fil.ion.ud.ac.uk/spm/). For single subject analysis, all images were realigned to the first volume, corrected for motion artifacts, coregistered with the participants' corresponding anatomical ( $\mathrm{T}_{1}$-weighted) images, normalized into standard stereotaxic space $(2 \times 2 \times 2 \mathrm{~mm}$ for the EPI, $1 \times 1 \times 1 \mathrm{~mm}$ for the 3D anatomical image), and smoothed using a $10-\mathrm{mm}$ full width at half-maximum Gaussian kernel. Applying a boxcar model, adjusted mean images (convolved with the modeled hemodynamic response and eliminating low-frequency noise) were computed for each condition and each participant in the context of SPM99. Activated voxels were identified by the General Linear Model approach (Friston et al., 1994). To test hypotheses about regionally specific condition effects for each stimulus type, the following linear contrasts were defined: 


\section{TABLE 1}

Means and SD for Reaction Times and Percentages of Correct Answers in All Conditions

\begin{tabular}{lccccc}
\hline & \multicolumn{2}{c}{ Hands } & & \multicolumn{2}{c}{ Tools } \\
\cline { 2 - 3 } \cline { 5 - 6 } Reaction time & Control & Experiment & & Control & Experiment \\
\hline Mean & 2115 & 2611 & & 2073 & 2783 \\
SD & 676 & 683 & & 672 & 670 \\
\% correct & & & & \\
$\quad$ Mean (SD) & $93(9)$ & $82(12)$ & & $91(6)$ & $75(13)$ \\
\hline
\end{tabular}

(1) $\mathrm{EH}>\mathrm{CH}$; (2) $\mathrm{ET}>\mathrm{CT}$. To determine specific activation in the control condition, we also contrasted the control minus experimental condition: (3) $\mathrm{CH}>$ $\mathrm{EH}$; (4) CT > ET. Finally, we contrasted the experimental conditions with each other for direct comparison of the stimulus type effect in the rotation conditions: (5) $\mathrm{EH}>\mathrm{ET}$; (6) $\mathrm{ET}>\mathrm{EH}$. The resulting set of voxel values for each contrast constitutes a statistical parametric map (SPM) of the T statistic (SPM $\{T\}$ ). These single subject contrast images were used for generating group statistical parametric maps ("random effects procedure") using a one-sample t test. The statistical height threshold used in the analysis of the random effects was set to $P<0.0001$ ( $T \geq 5,45$, uncorrected). The activated voxels surviving these procedures were superimposed on the averaged stereotaxically normalized high-resolution MR-anatomical scans of the 12 subjects in order to identify the anatomical regions. Coordinates in SPM were adjusted to Talairach and Tournoux (1988) atlas brain coordinates using the Meyer-Lindenberg conversion (with $X^{\prime}=$ $0.88 X-0.8 ; Y^{\prime}=0.97 Y-3.32 ; Z^{\prime}=0.05 Y+0.88 Z-$ 0.44) as described by $M$. Brett (URL http://www. mrc-cbu.cam.ac.uk/l maging/mnispace.html).

\section{RESULTS}

\section{Behavioral Data}

Responses and response times were recorded during the experiment, which allowed us to check whether the behavioral signature of mental rotation was present in the participants' performance. The behavioral signature of mental rotation implies that reaction times become longer in a linear fashion when the angular disparity between the objects increases. We trimmed response times before analysis by considering reaction times lower than 500 ms as answers of the previous trial because no latency between the trials existed and participants sometimes accidentally pressed to answer the previous trial when the next trial was already presented. This procedure was applied to $1.7 \%$ of all data. Means and error rates for all conditions are shown in Table 1.
Figure 2 shows mean reaction time (RT) as a function of angular disparity for all stimulus types. Separate univariate one-way ANOVAs for repeated measurements were performed for hands and tools with RT as a dependent variable and angular disparity and participant as random factors. These ANOVAs reveal a main effect for angular disparity both in the hands condition $\left(F^{\prime}=4.09 ; \mathrm{df}=3,33.73, \mathrm{P}<0.015\right)$ and in the tools condition $\left(F^{\prime}=5.94\right.$; $\left.d f=3,34.32, P<0.005\right)$. There were no significant interactions between angular disparity and participant. In a further step, we performed trend analysis to check for linearity in both conditions. There was a significant linear trend for angular disparity in the tools condition $(P=0.010$; $\left.\mathrm{ETA}^{2}=0.018\right)$. No significant higher order trend was present. In the hands condition, a linear trend failed to reach statistical significance $(P=0.097)$, while there was a significant cubic trend $\left(P=0.014 ; \mathrm{ETA}^{2}=\right.$ 0.016).

\section{Imaging Data}

Table 2 and Fig. 3 summarize significant peak hemodynamic responses for each contrast.

\section{Superior Parietal Cortical Regions}

Activation of the SPL (with activation sometimes spreading to the IPS) was clearly and bilaterally present during mental rotation of hands and tools relative to their control conditions. In the tools condition, SPL activation was more profound in the left hemisphere, as could also be determined from the ET $>\mathrm{EH}$ contrast. In the control > experimental conditions no SPL activation was observed.

\section{Extrastriate Visual Regions}

The right fusiform gyrus (BA 18) was activated during the mental rotation of hands. In the tools condition, bilateral activation of the superior occipital gyrus (BA 19) was found. Direct comparison of both rotation tasks

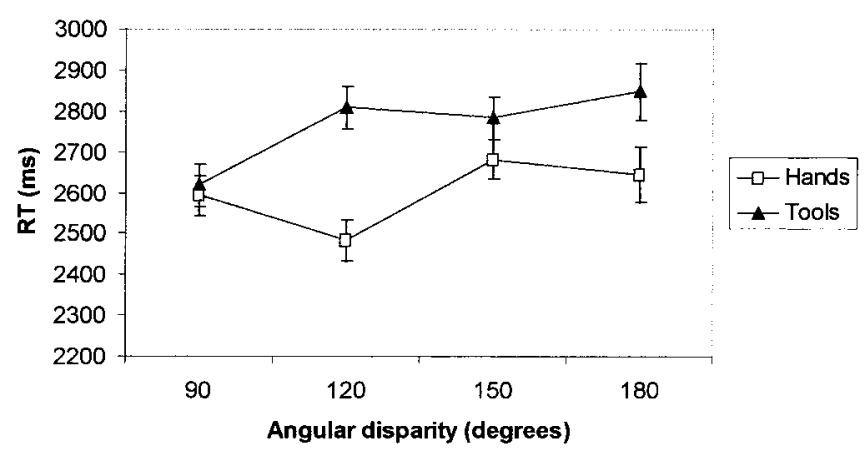

FIG. 2. Reaction times (RTs; means $\pm S E M$ ) measured in the two experimental conditions. A significant linear trend is present in the tools condition, while a significant nonlinear trend is present in the hands condition. 
TABLE 2

Foci of Significant Activation and Their Stereotaxic Coordinates for All Contrasts

\begin{tabular}{|c|c|c|c|c|c|c|c|}
\hline \multirow[b]{2}{*}{ Contrast } & \multirow[b]{2}{*}{ Anatomical region } & \multirow[b]{2}{*}{ BA } & \multirow[b]{2}{*}{ Hemisphere } & \multirow[b]{2}{*}{ Maximum Z score } & \multicolumn{3}{|c|}{ Coordinates } \\
\hline & & & & & $x$ & y & z \\
\hline \multirow[t]{6}{*}{$\mathrm{EH}>\mathrm{CH}$} & Cerebellum & & $\mathrm{R}$ & 4.64 & 17 & -89 & -19 \\
\hline & Superior parietal lobule (SPL) & 7 & $\mathrm{~L}$ & 4.82 & -25 & -48 & 31 \\
\hline & Superior parietal lobule (SPL) & 7 & $\mathrm{R}$ & 5.00 & 29 & -50 & 50 \\
\hline & Precentral sulcus (premotor) & 6 & L & 4.64 & -24 & -9 & 50 \\
\hline & Precentral sulcus (premotor) & 6 & $\mathrm{R}$ & 5.12 & 26 & -9 & 52 \\
\hline & Fusiform gyrus & 18 & $\mathrm{R}$ & 4.36 & 33 & -85 & -12 \\
\hline \multirow{9}{*}{$\mathrm{ET}>\mathrm{CT}$} & Pons & & $\mathrm{L}$ & 5.21 & -4 & -31 & -25 \\
\hline & Pons & & $\mathrm{R}$ & 4.56 & 1 & -40 & -27 \\
\hline & Cerebellum & & $\mathrm{L}$ & 5.00 & -24 & -69 & -23 \\
\hline & Cerebellum & & $\mathrm{R}$ & 5.19 & 24 & -60 & -32 \\
\hline & Superior parietal lobule (SPL) & 7 & $\mathrm{~L}$ & 4.95 & -29 & -58 & 53 \\
\hline & Superior parietal lobule (SPL) & 7 & $\mathrm{R}$ & 4.38 & 17 & -67 & 48 \\
\hline & Precentral sulcus (premotor) & 6 & $\mathrm{~L}$ & 4.59 & -25 & -3 & 51 \\
\hline & Superior occipital gyrus & 19 & L & 4.88 & -22 & -79 & 22 \\
\hline & Superior occipital gyrus & 19 & $\mathrm{R}$ & 4.79 & 24 & -81 & 25 \\
\hline \multirow{4}{*}{$\mathrm{CH}>\mathrm{EH}$} & Medial temporal gyrus & 21 & $\mathrm{R}$ & 5.23 & 59 & -36 & -9 \\
\hline & Superior frontal gyrus & 8 & $\mathrm{R}$ & 5.05 & 6 & 43 & 44 \\
\hline & Precuneus & 19 & L & 4.33 & -4 & -69 & 30 \\
\hline & Cingulate gyrus & 31 & $\mathrm{~L}$ & 4.23 & -3 & -36 & 33 \\
\hline \multirow{6}{*}{$\mathrm{CT}>\mathrm{ET}$} & Cingulate gyrus & 23 & L & 5.06 & -1 & -27 & 27 \\
\hline & Medial temporal gyrus & 39 & $\mathrm{R}$ & 5.02 & 48 & -63 & 16 \\
\hline & Medial temporal gyrus & 21 & $\mathrm{~L}$ & 5.01 & -50 & -3 & -7 \\
\hline & Superior frontal gyrus & 9/10 & L & 4.83 & -8 & 57 & 15 \\
\hline & Superior frontal gyrus & 8 & $\mathrm{R}$ & 4.71 & 4 & 37 & 49 \\
\hline & Angular gyrus & 39 & L & 4.48 & -47 & -60 & 34 \\
\hline \multirow[t]{3}{*}{$\mathrm{EH}>\mathrm{ET}$} & Lingual gyrus & 18 & $\mathrm{R}$ & 5.03 & 10 & -87 & -5 \\
\hline & Medial temporal gyrus & 39 & $\mathrm{R}$ & 4.96 & 45 & -63 & 9 \\
\hline & Inferior temporal gyrus & 37 & $\mathrm{~L}$ & 4.41 & -40 & -69 & 1 \\
\hline \multirow[t]{3}{*}{$\mathrm{ET}>\mathrm{EH}$} & Lingual gyrus & 19 & $\mathrm{R}$ & 5.03 & 17 & -62 & 2 \\
\hline & Cerebellum & & L & 4.64 & -29 & -54 & -15 \\
\hline & Superior parietal lobule & 7 & $\mathrm{~L}$ & 4.51 & -29 & -62 & 53 \\
\hline
\end{tabular}

revealed predominant right hemispheric occipital cortex activation in slightly different regions. Rotation of hands showed increased bilateral temporal (middle and inferior gyri) over rotations of tools. The control conditions also revealed stronger activation in visual association areas, particularly of the right middle temporal gyrus.

\section{Motor and/or Premotor Regions}

An area that was found to be activated in both rotation paradigms was the cerebellum. The ET $>\mathrm{EH}$ contrast further revealed that (left) cerebellar activity was greater during the mental rotation of tools. A striking difference between both rotations tasks was the involvement of premotor regions. The premotor cortex was bilaterally activated in the hands condition. In the tools condition, however, only left hemisphere premotor activation was registered. Direct contrast of both rotation tasks revealed no extra premotor activity of one task over the other. The control conditions revealed additional involvement of the frontal oculomotor regions in the superior frontal gyrus.

\section{Other Cortical Areas}

Posterior cingulate activation was detected in both control conditions relative to the rotation conditions. The control tools condition additionally activated left hemispheric prefrontal and angular regions.

\section{DISCUSSION}

\section{Behavioral Data}

When looking at the behavioral data, notable differences can be seen between the two types of stimuli. While participants were clearly able to perform both tasks about equally well (82 and $75 \%$ correct for hands and tools, respectively), the reaction time profiles do not obey to the same rule. In the case of the mental rotation of tools, reaction times increase linearly (judging from the statistically significant linear trend), which corresponds to the classical finding of linearly increasing reaction times with increasing angular disparity and validates the claim that mental rotation was performed. 
$\mathbf{E H}>\mathbf{C H}$
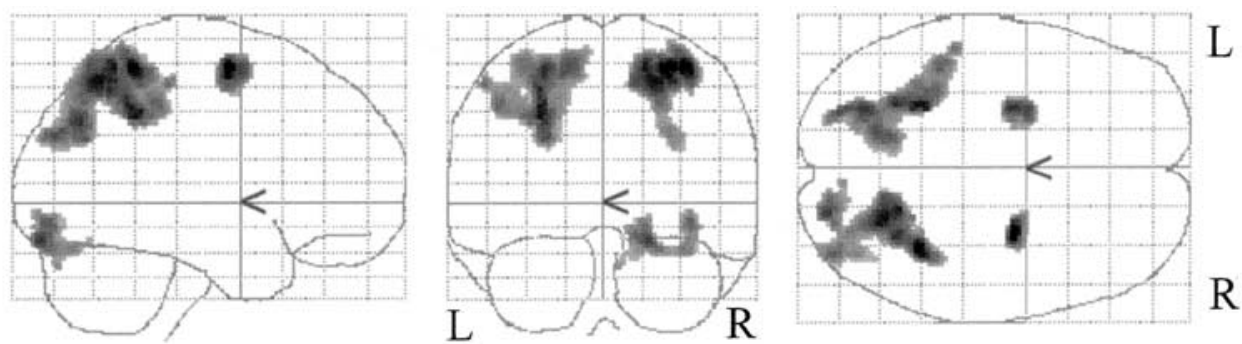

$\mathbf{E T}>\mathbf{C T}$
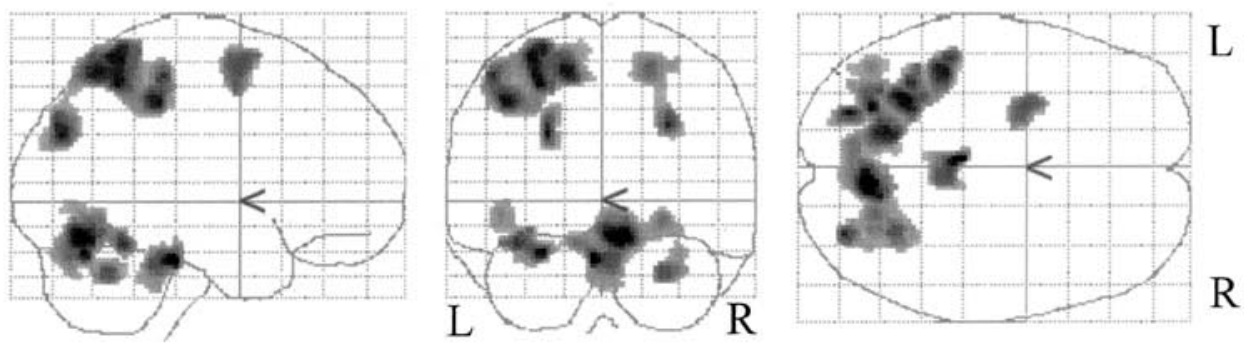

$\mathbf{C H}>\mathbf{E H}$
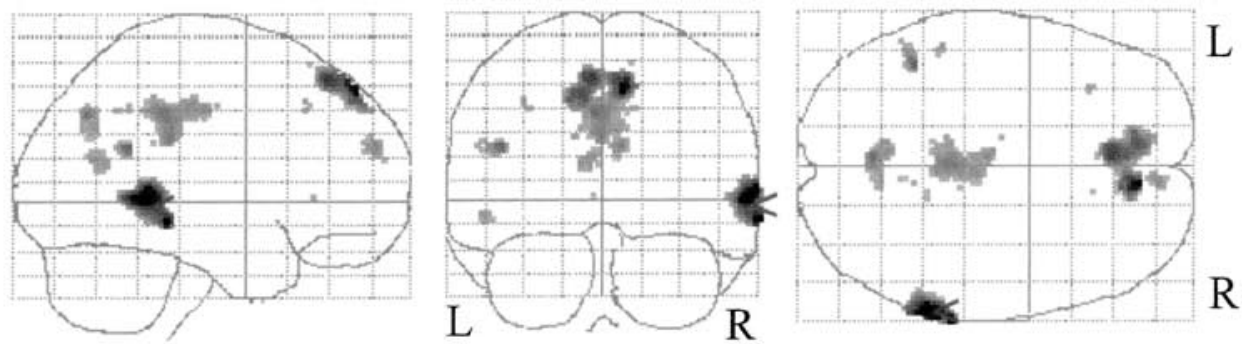

CT $>$ ET
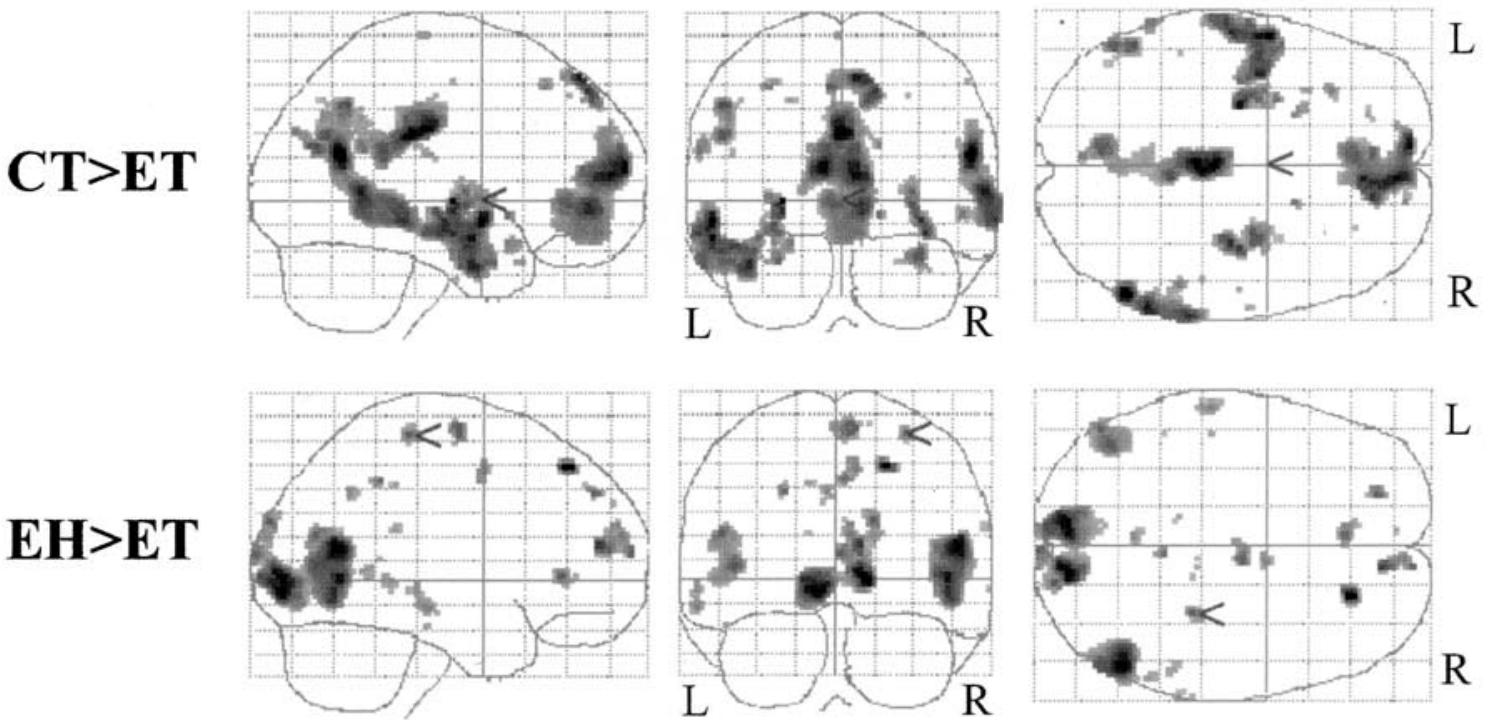

$\mathbf{E T}>\mathbf{E H}$
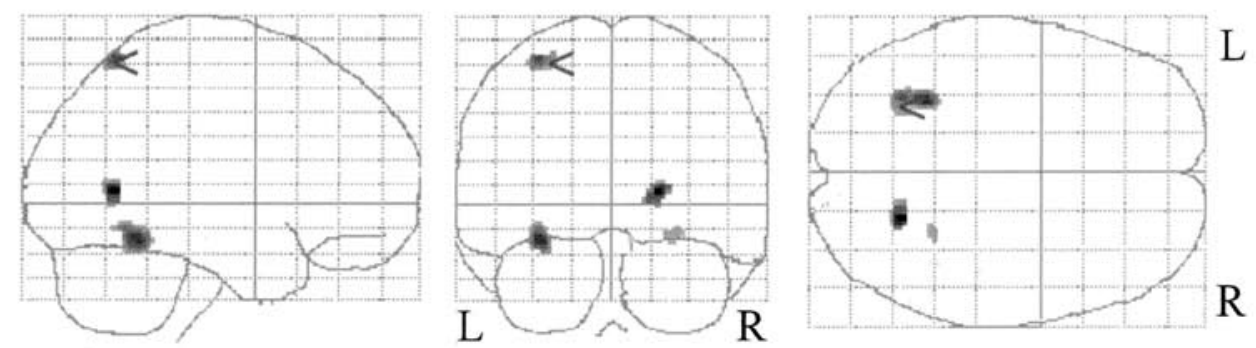

FIG. 3. Significant areas of activation for the designated contrasts. C, E, H, and T as defined in the legend to Fig. 1. 
The profile of reaction times in the hand condition does not obey linearity, however. Although this at first may seem to be in contradiction with the notion of mental rotation, it is actually supportive of the claim that mental rotation was performed in the hand condition. The nonlinear behavior of the reaction times in mental rotation with hands as the stimulus type can namely be explained by looking at the results of the earlier work of Parsons (1994), in which he examined the reaction time profiles of participants who mentally rotated figures of hands while the orientation of hands was manipulated (e.g., view from the back of the hand, view from the palm of the hand, etc.). Results show that reaction time profiles strongly deviate from the classical linear pattern in some instances because some orientations are harder to imagine due to their awkward posture. There were too few trials in this study to disentangle all different postures (palm vs back), hands (left vs right), and angular disparities, but it is plausible that the effect that Parsons described exists in our study as well, causing the nonlinearity in the reaction time profile for the hands condition.

\section{I maging Data}

In regard to the neural domain we hypothesized that both stimulus types partially share a common neural substrate, namely the involvement of the superior parietal cortex and that preparatory motor processes would be recruited when participants needed to mentally rotate pictures of hands or tools. A partially common neural substrate was found for hands and tools: bilateral activation of the SPL (BA 7). Involvement of SPL (extending to IPS and sometimes IPL) is almost consistently found in brain imaging studies examining mental rotation. As suggested by the experimental minus control contrasts, and confirmed by the ET $>\mathrm{EH}$ contrast, left SPL activation is more substantial in the tools condition. This is in agreement with the involvement of the posterior parietal cortex for goal-directed motor behavior (and apraxia) and its recruitment whenever an action involves objects (Freund, 2001). Our results also contribute to the hypothesis of a left hemispheric lateralization of the command system underlying voluntary object-related praxis (Choi et al., 2001; Moll et al., 2000).

Activation of secondary visual areas (BA 18 or 19) was found in both rotation conditions, which is also in agreement with previous research that believed it to reflect the processing of object movement (Cohen et al., 1996). The secondary contrasts $(\mathrm{EH}>\mathrm{ET}$ and ET $>$ $E H)$ reveal more pronounced activation in the occipital and temporal visual association areas in the hands condition. This could reflect the necessity of increased perceptual processing of the hands stimuli because they are visually more complex and/or visually less differentiated from each other than the tools stimuli.
Subtracting the experimental conditions from the control conditions revealed enhanced activity in the frontal eye fields, temporal visual association areas, and posterior cingulate cortex. This seems to suggest the use of a different and probably more visual strategy based on visual comparison of the stimuli during the control tasks.

Both rotation conditions also elicited activation of the cerebellum, known to be active during the planning and execution of movement (Posner and Petersen, 1990). Cerebellar activation is frequently found during covert actions (J eannerod, 2001). Parsons et al. (1995) and Bonda et al. (1995) have also found cerebellar activation in a mental rotation study using hands as stimuli. Again, the experimental minus control contrasts suggested, and the ET $>\mathrm{EH}$ confirmed, greater cerebellar activation in the tools condition. Tool-userelated activities in the monkey revealed (among other areas) bilateral or ipsilateral activation in the cerebelIum (Obayashi et al., 2001). The cerebellar activity was interpreted to aid in the storage or retrieval of longterm memory for tool use learning or reconstruction of the required body images. I psilateral cerebellar activity in humans was also observed during the perception of objects (Grèzes and Decety, 2002). According to these authors, the ipsilateral cerebellum, premotor cortex, and left parietal lobe are involved during the perception of objects when subjects prepare to act and when they imagine acting upon them. Although there was a trend toward bilateral cerebellar activation, that of the left cerebellar (presumed contralateral) was clearly more robust in our study.

Imaging data show a surprising difference in the activation of premotor areas between the rotation paradigms: in the hands condition the premotor area was bilaterally activated while in the tools condition only the left hemisphere premotor area was activated. Because in the hands rotation task both left hands and right hands were used as stimuli, the results agree with the hypothesis that participants imagined the pictures as their own left and right hands and mentally moved their own hands in order to find out whether stimuli were same or mirror images. In the tools condition only left hemisphere premotor activation, responsible for the planning of motor actions of the right half of the body, was found. Because all participants were consistent right-handers, this supports the same hypothesis of imagining motor action: participants mentally grasped and rotated tools to see if they were same or mirror images. Direct contrast of the rotation conditions $(\mathrm{EH}>\mathrm{ET})$ did not reveal an expected region of significant right premotor activity. This suggests to us that the mental rotation of tools, just like hands, elicits at least some bilateral premotor activation. Because the premotor activity of the right hemisphere is much smaller in the tools condition, it does not reach significance in comparison with its control condition. It 
is, however, sufficient to neutralize the lateralized difference when contrasted directly with the experimental hands condition.

Our findings correspond well with the simulation theory put forward by J eannerod (2001) in which he postulates that the same neural mechanisms are activated during covert action (such as imagined action) as during overt executed action. The results suggest that in our mental rotation tasks two slightly different covert actions took place: imagining stimuli as one's own hands and moving them (in the hands condition) and imagining grasping an object and moving it (in the tools condition). These covert actions of motor imagery appear to mimic the "natural way" in which a person would manipulate the object in reality. Recent research confirms the idea of an involvement of motor representation al ready during the perception of objects and that the perception of objects automatically potentiates the actions they afford, even in the absence of explicit intention to act (Grèzes and Decety, 2002). Here, too, right-handed subjects activate left hemispheric motor areas when confronted with color photographs of real graspable objects even if the task does not involve motor imagery. It appears from our results that the activation of cortical regions during mental rotation is at least in part determined by an automatic activation that depends on the actions elicited by the kind of stimuli presented. This automatic or intrinsic bottom-up process can be surpassed by voluntary topdown control through the manipulation of explicit strategies, as was demonstrated in the Kosslyn et al. study (2001b).

Since premotor activation is much more frequently reported than primary motor activation in mental rotation research, we might ask under what circumstances the primary motor cortex is recruited during motor imagery? In contrast with premotor cortex, M1 appears to be not very reactive during motor imagery; it amounts to less than one-third of the level of activation observed during motor execution and may not be found in all subjects (Gerardin et al., 2000; J eannerod, 2001). Premotor areas are believed to play a critical role in motor planning, whereas $\mathrm{M} 1$ is considered responsible for motor execution. Almost every mental rotation study that reports primary motor cortex activation considered overt movement (button press artifacts, priming effects, or subtle twitching movements to guide internal processing) as a possible explanation of the findings. Most studies come to the conclusion that these methodological remarks do not sufficiently explain the significant primary motor cortex activation (Kosslyn et al., 2001b; Vingerhoets et al., 2001). Moreover, transcranial magnetic stimulation over M1 during the mental rotation of hands does disrupt processing, suggesting that the $\mathrm{M} 1$ area plays a functional role in mental rotation (Ganis et al., 2000). Kosslyn et al. (2001b) hypothesized that M1 is partially activated in the baseline condition as well because subjects imagine mentally moving one figure over until it covers the other. The motor processes involved in the baseline condition could obscure activation in $\mathrm{M} 1$ in the mental rotation condition when both are compared. Indeed, in their study M1 activation was found only when the results from the endogenous and exogenous conditions were directly compared and not when these conditions were compared to the same baseline. Since our data do not reveal MI activation when each rotation condition is compared with its appropriate baseline condition, a similar mechanism can be hypothesized. The fact that premotor cortex activation is much less frequently obscured by this potential mechanism (we can expect premotor activity in the baseline condition as well) suggests an enhanced recruitment of premotor areas over that of primary motor areas during mental rotation. Why would this be the case? Analogous to parietofrontal circuits for action and space perception in the monkey brain (Matelli and Luppino, 2001), a strong connection between the posterior parietal and premotor cortex in humans can be hypothesized. Dorsal premotor areas of the macaque monkey are intimately connected with posterior parietal areas and respond to somatosensory, in particular proprioceptive, stimuli and are believed to integrate information about the target location and the arm to be used to plan a forthcoming action (Fogassi et al., 1999; Hoshi and Tanji, 2000). This area was also activated preferentially during incorporated tool use (e.g., the tool as part of the "body image") in the macaque monkey (Obayashi et al., 2001). The hypothesis of a similar connection in humans is supported by a comparable functional specialization of the homologous dorsal premotor area in the human brain (Boussaoud, 2001), by the involvement of the human premotor cortex in dynamic visuospatial imagery (Lamm et al., 2001), and by the fact that mental rotation studies reporting posterior parietal activation almost invariably also report premotor cortex activation. The association between primary motor activation and posterior parietal cortex activation is less frequently found. These findings suggest that both primary motor and premotor regions are active during mental rotation, but that activation is more prominent in the premotor areas, mainly because of high posterior parietal input to spatially guide a potential movement. The feeble yet existing anticipatory $\mathrm{M} 1$ activity is easily obscured by methodological "noise" and frequently does not reach statistical significance.

We conclude that our results show that how mental rotation is performed in the human brain depends to a certain extent on what is mentally rotated. It is striking that the mental rotation of the left and right hands elicits bilateral premotor activation while the mental rotation of tools (frequently manipulated with the right hand in right-handers) yields significant left hemisphere premotor activation only. Other studies that 
used more abstract stimuli did not find any (pre)motor activation at all. Why would there not be a single solving strategy in the brain that dealt with all kinds of stimuli? Behavioral data give us a hint of why these differences may exist. Mental rotation of hands and tools appears to be much faster and less error prone than the more abstract mental rotation of ShepardMetzler cubes reported in other studies (Carpenter et al., 1999; K osslyn et al., 1998). It thus seems that being able to use the internal strategy, to imagine holding or moving a stimulus, gives participants a big advantage over when the stimuli do not lend themselves to this strategy and participants are therefore forced to use the external strategy. The present results are not conclusive as far as the external strategy is concerned. It would be interesting to contrast abstract and not easy to grasp but less difficult shapes against the hands and tools stimuli to see if motor processes would be absent in these stimuli. Future research also could focus on objects that are manipulated by two hands (e.g., a steering wheel), which should engender bilateral premotor activation just as the hands stimuli did in the present experiment. Also of interest would be to examine the mental rotation of tools in left-handers by the use of $\mathrm{fMRI}$ to see if this task would lead to right hemisphere premotor activation. All these lines of research could bring us further to the notion that imaginative functions such as mental rotation can imply the same neural mechanisms as the actions that are imagined.

\section{REFERENCES}

Alivesatos, B., and Petrides, M. 1997. Functional activation of the human brain during mental rotation. Neuropsychologia 35: 111118.

Barnes, J ., Howard, R. J ., Senior, C., Brammer, M., Bullmore, E. T., Simmons, A., Woodruff, P., and David, A. S. 2000. Cortical activity during rotational and linear transformations. Neuropsychologia 38: 1148-1156.

Bonda, E., Petrides, M., Frey, S., and Evans, A. 1995. Neural correlates of mental transformations of the body-in-space. Proc. Natl. Acad. Sci. USA 92: 11180-11184.

Boussaoud, D. 2001. Attention versus intention in the primate motor cortex. Neurol mage 14: S40-S45.

Carpenter, P. A., J ust, M. A., Keller, T. A., Eddy, W., and Thulborn, K. 1999. Graded functional activation in the visuospatial system with the amount of task demand. J . Cognit. Neurosci. 11: 9-24.

Choi, S. H., Na, D. L., Kang, E., Lee, K. M., Lee, S. W., and Na, D. G. 2001. Functional magnetic resonance imaging during pantomiming tool-use gestures. Exp. Brain Res. 139: 311-317.

Cohen, M. S., Kosslyn, S. M., Breiter, H. C., Digirolamo, G. J ., Thompson, W. L., Anderson, A. K., Bookheimer, S. Y., Rosen, B. R., and Belliveau, J. W. 1996. Changes in cortical activity during mental rotation. A mapping study using functional MRI. Brain 119: $89-100$.

deSperati, C., and Stucchi, N. 1997. Recognizing the motion of a graspable object is guided by handedness. NeuroReport 8: 27612765.

Fogassi, L., Raos, V., Franchi, G., Gallese, V., Luppino, G., and Matelli, M. 1999. Visual responses in the dorsal premotor area F2 of the macaque monkey. Exp. Brain Res. 128: 194-199.
Freund, H.-J . 2001. The parietal lobe a s a sensorimotor interface: A perspective from clinical and neuroimaging data. Neurol mage 14: S142-S146.

Friston, K. J ., Worsley, K. J ., Frackowiak, R. S. J ., Maziotta, J . C., and Evans, A. C. 1994. Assessing the significance of focal activations using their spatial extent. Hum. Brain Mapping 1: 210-220.

Ganis, G., Keenan, J . P., Kosslyn, S. M., and Pascual-Leone, A. 2000. Transcranial magnetic stimulation of primary motor cortex affects mental rotation. Cereb. Cortex 10: 175-180.

Georgopoulos, A. P., Crutcher, M. D., and Schwartz, A. B. 1989. Cognitive spatial-motor processes 3: Motor cortical prediction of movement direction during an instructed delay period. Exp. Brain Res. 75: 183-194.

Gerardin, E., Sirigu, A., Lehéricy, S., Poline, J .-B., Gaymard, B., Marsault, C., Agid, Y., and Le Bihan, D. 2000. Partially overlapping neural networks for real and imagined hand movements. Cereb. Cortex 10: 1093-1104.

Grèzes, J ., and Decety, J . 2002. Does visual perception of object afford action? Evidence from a neuroimaging study. Neuropsychologia 40: 212-222.

Harris, I. M., Egan, G. F., Sonkkila, C., Tochon-Danguy, H. J ., Paxinos, G., and Watson, J. D. G. 2000. Selective right parietal lobe activation during mental rotation. A parametric PET study. Brain 123: 65-73.

Hoshi, E., and Tanji, J . 2000. Integration of target and body-part information in the premotor cortex when planning action. Nature 408: $466-470$.

Howard, R. J ., Bullmore, E., Brammer, M., Williams, S. C., Mellers, J ., Woodruff, P., David, A., Andrew, C., Allin, M., Simmons, A., and Cox, T. 1995. Activation of area V5 by visual perception of motion demonstrated with echoplanar MR imaging. Magn. Reson. Imaging 13: 907-909.

I waki, S., Ueno, S., Imada, T., and Tonoike, M. 1999. Dynamic cortical activation in mental image processing revealed by biomagnetic measurement. NeuroReport 10: 1793-1797.

J eannerod, M. 2001. Neural simulation of action: A unifying mechanism for motor cognition. Neurol mage 14: S103-S109.

J ordan, K., Heinze, H. J ., Lutz, K., Kanowski, M., and J äncke, L. 2001. Cortical activations during the mental rotation of different visual objects. Neurol mage 13: 143-152.

Kosslyn, S. M. 1994. Image and Brain. MIT Press, Cambridge, MA.

Kosslyn, S. M., Digirolamo, G. J ., Thompson, W. L., and Alpert, N. M. 1998. Mental rotation of objects versus hands: Neural mechanisms revealed by positron emission tomography. Psychophysiology 35: 151-161.

Kosslyn, S. M., Ganis, G., and Thompson, W. L. 2001a. Neural foundations of imagery. Nat. Rev. Neurosci. 2: 635- 642.

Kosslyn, S. M., Thompson, W. L., Wraga, M., and Alpert, N. A. 2001b. I magining rotation by endogenous versus exogenous forces: Distinct neural mechanisms. NeuroReport 12: 2519-2525.

Lamm, C., Windischberger, C., Leodolter, U., Moser, E., and Bauer, H. 2001. Evidence for premotor cortex activity during dynamic visuospatial imagery from single-trial functional magnetic resonance imaging and event-related slow cortical potentials. NeuroImage 14: 268-283.

Matelli, M., and Luppino, G., 2001. Parietofrontal circuits for action and space perception in the macaque monkey. Neurol mage 14: S27-S32.

Moll, J ., de Oliveira-Souza, R., Passman, L. J ., Cunha, F. C., SouzaLima, F., and Andreiuolo, P. A. 2000. Functional MRI correlates of real and imagined tool-use pantomimes. Neurology 54: 13311336.

Obayashi, S., Suhara, T., Kawabe, K., Okauchi, T., Maeda, J ., Akine, 
Y., Onoe, H., and Iriki, A. 2001. Functional brain mapping of monkey tool use. Neurol mage 14: 853-861.

Oldfield, R. C. 1971. The assessment and analysis of handedness: The Edinburgh inventory. Neuropsychologia 9: 97-113.

Parsons, L. M. 1994. Temporal and kinematic properties of motor behavior reflected in mentally simulated action. J. Exp. Psychol. Hum. Percept. Performance 20: 709-730.

Parsons, L. M., Fox, P. T., Downs, J. H., Glass, T., Hirsch, T. B., Martin, C. C., J erabek, P. A., and Lancaster, J. L. 1995. Use of implicit motor imagery for visual shape discrimination as revealed by PET. Nature 375: 54-58.

Posner, M. I., and Petersen, S. E. 1990. The attention system of the human brain. Ann. Rev. Neurosci. 11: 1325-1342.

Pylyshyn, Z. W. 1973. What the mind's eye tells the mind's brain: A critique of mental imagery. Psychol. Bull. 80: 1-24.

Pylyshyn, Z. W. 1981. The imagery debate: Analogue media versus tacit knowledge. Psychol. Rev. 88: 16- 45.

Richter, W., Somorjai, R., Summers, R., and J armasz, M. 2000. Motor area activity during mental rotation studied by time-resolved single-trial fMRI. J . Cognit. Neurosi. 12: 310-320.
Richter, W., Ugurbil, K., Georgopoulos, A., and Kim, S.-G. 1997. Timeresolved fMRI of mental rotation. NeuroReport 8: 36973702.

Shepard, R. N., and Cooper, L. A. 1982. Mental Images and Their Transformations. MIT Press, Cambridge, MA.

Shepard, R. N., and Metzler, J. 1971. Mental rotation of threedimensional objects. Science 171: 701-703.

Tagaris, G. A., Kim, S. G., Strupp, J . P., Andersen, P., U gurbil, K., and Georgopoulos, A. P 1996. Quantitative relations between parietal activation and performance in mental rotation. NeuroReport 7: $773-776$.

Talairach, J ., and Tournoux, P. 1988. Co-planar Stereotaxic Atlas of The Human Brain. 3-Dimensional Proportional System: An Approach to Cerebral Imaging. Thieme, New York.

Vingerhoets, G., Santens, P., Van Laere, K., Lahorte, P., Dierckx, R. A., and De Reuck, J. 2001. Regional brain activity during different paradigms of mental rotation in healthy volunteers: $A$ positron emission tomography study. Neurol mage 13: 381-391.

Wexler, M., Kosslyn, S. M., and Berthoz, A. 1998. Motor processes in mental rotation. Cognition 68: 77-94. 